\title{
Intensity discrimination and loudness for tones in broadband noise
}

\author{
BRUCE A. SCHNEIDER \\ University of Toronto, Mississauga, Ontario, Canada \\ and \\ SCOTT PARKER \\ The American University, Washington, DC
}

\begin{abstract}
In two previous papers (Parker \& Schneider, 1980; Schneider \& Parker, 1987), we developed a model, based on Fechner's assumption, which successfully predicted the relationship between loudness and intensity discrimination for tones presented in quiet and in notched noise. In the present paper, pure-tone intensity-increment thresholds and loudness matches were determined for several levels of a standard tone in the presence of a broadband masker whose spectrum level was set to $35 \mathrm{~dB}$ below that of the standard tone. The model was unable to relate loudness to intensity discrimination under these conditions. Thus, the spectral composition of the masker affects the relationship between loudness and intensity discrimination in ways that cannot be accounted for by the model.
\end{abstract}

In a previous paper (Schneider \& Parker, 1987), we examined the relationship between intensity discrimination and loudness for tones in notched noise and developed a model in which the effects of notched noise on discrimination performance were linked to the effects of notched noise on loudness. This model incorporated Fechner's notion that differences noticed equally often are subjectively equal. We proposed that the loudness difference between the standard and comparison stimuli $\left(I_{s}, I_{c}\right)$ at the difference threshold was constant; that is, $\Delta L=L_{c}-L_{\mathrm{s}}$ $=k$ for all values of $I_{\mathrm{s}}$, where $\Delta L$ is the constant loudness increment corresponding to the JND. If loudness is a power function of intensity, then at threshold we have $I_{\mathrm{c}}^{p^{\prime}}-I_{\mathrm{s}}^{p^{\prime}}=k$. We used $p^{\prime}$ as the symbol for the discrimination exponent to allow for the possibility that it might differ from the exponent found in other loudness-judgment paradigms. In quiet, the value of $p^{\prime}$ was found to be approximately .07 for sound intensity (Parker \& Schneider, 1980); in notched noise, the value of the exponent depended on the extent to which the noise reduced tonal loudness. Specifically, we found that a model in which $p^{\prime}=.07 R$, where $R$ is the ratio of the loudness of the tone in notched noise to its loudness in quiet, provided a good fit to the data of 7 subjects and to their average data. Note that when tones are presented in quiet, there is no loudness reduction, so $R=1$ and $p^{\prime}=.07$. Thus,

This work was supported by the Natural Sciences and Engineering Research Council of Canada. We would like to thank Jane Carey and Sandra Piasecki for their assistance in conducting these experiments. Requests for reprints should be sent to Bruce Schneider, Department of Psychology, Erindale Campus, University of Toronto, Mississauga, Ontario L5L 1C6, Canada. this model accounts for pure-tone intensity discrimination in quiet as a limiting case.

In computing the amount of loudness reduction in this model, we assumed that loudness was a power function of intensity $L=\mathrm{I}^{p}$, where $p$ is the exponent for loudness in quiet and not to be confused with $p^{\prime}$, the discrimination exponent. In fitting this model we were able to obtain estimates of the loudness exponents, $p$, for the growth of loudness in quiet for each of our 7 subjects. These estimates ranged from .17 to .40 , a range that is consistent with data obtained from direct estimates of loudness (Marks, 1974). We found this consistency encouraging.

A reasonable test of the generality of the model is to see whether or not it can account for the relationship between intensity discrimination and masking for other types of noise. In this paper, we report the results of testing this relationship in broadband noise rather than notched noise with the 3 subjects from Schneider and Parker (1987) who were still available for testing.

\section{METHOD}

\section{Subjects}

Three of the subjects (L.T., R.L., and T.T.) who served in the Schneider and Parker (1987) experiment participated in this study. All were female; their ages ranged from 21 to 33 years. None of the 3 had any known auditory pathology.

\section{Apparatus and Stimuli}

The masker was a bandpassed Gaussian noise whose spectrum was flat up to $5 \mathrm{kHz}$ and declined by $30 \mathrm{~dB}$ per octave thereafter. The $1-\mathrm{kHz}$ pure-tone signals were produced by a programmable function generator (Hewlett-Packard 3325A) and had 10-msec rise and decay times. Programmable attenuators were used to set the noise level and the level of the standard tone. The spectrum level of the noise was always set $35 \mathrm{~dB}$ below the intensity of the stan- 
dard tone, and thus rose and fell with the intensity of the standard tone. The five standard tone intensities were $30,44,57,71$, and $84 \mathrm{~dB}$. Stimuli were presented over TDH-49 earphones. Operations and timing were managed by a Commodore computer.

For intensity discrimination, a set of five comparison-tone intensities was used for each standard intensity. In most cases, the set of comparison tones included intensities that exceeded the standard intensity by $.5,1,1.5,2$, and $2.5 \mathrm{~dB}$. When necessary, the loudest of these was replaced by a tone whose intensity exceeded the standard by $.25 \mathrm{~dB}$.

\section{Procedure}

Loudness matching. The subject's task was to match the loudness of the $1-\mathrm{kHz}$ tone presented in quiet to the loudness of the tone in the noise. Both the tone in noise and the tone in quiet were presented monaurally to the same ear. A trial consisted of alternate presentations of $750 \mathrm{msec}$ of tone in noise and $750 \mathrm{msec}$ of tone alone, separated by 750 -msec silent interstimulus intervals. A trial began with the intensity of the tone in quiet set randomly from 1 to $7 \mathrm{~dB}$ below that of the tone in noise. The subject controlled the intensity of the tone in quiet by pressing either of two buttons, one of which increased its intensity by $1 \mathrm{~dB}$ and the other of which decreased it by the same amount. When satisfied with the match, the subject pressed a third button to end the trial. The next trial began $750 \mathrm{msec}$ later. The intensity of the tone in the noise remained unchanged throughout the trial.

During a session, the five standard intensities of the tone in noise were presented five times each for a total of 25 trials per session. The five intensities were permuted randomly within each of the five blocks.

Each subject participated in six sessions with broadband masking noise, providing a total of $\mathbf{3 0}$ loudness matches at each standard tone intensity. The data from the first session were discarded; only the last 25 loudness matches were used in data analysis.

Intensity discrimination. Stimulus presentation was monaural. Each session consisted of $\mathbf{2 0 0}$ two-interval forced-choice trials involving one of the five standard $1-\mathrm{kHz}$ tones and one of the comparison intensities. The standard intensity was constant throughout the session. Standard and comparison tones appeared equally often in the first and second intervals in an otherwise random sequence, and the subject was instructed to indicate the interval containing the louder tone by pressing one of two buttons. Tone duration and interstimulus interval were both $750 \mathrm{msec}$. The subject pressed a button to initiate a trial. Feedback was provided by the illumination of one of two signal lights. Percentage correct was determined for each of the five comparison increments at each of the five standard intensities. The order of presentation of the 25 standard-comparison combinations was randomized for each subject. The pairs that bracketed $75 \%$ accuracy at each standard were retested. Threshold was the standard-comparison separation corresponding to $75 \%$ accuracy and was estimated by linear interpolation between the two bracketing increments. Best performances were used for the interpolations.

\section{RESULTS AND DISCUSSION}

Figure 1 plots the decibel level of a tone in quiet (averaged over the 3 subjects) that matched the loudness of a standard tone in broadband noise. Also plotted in Figure 1 is the corresponding function for tones in notched noise for these same 3 subjects (from Schneider \& Parker, 1987). Figure 1 shows that tonal loudness was reduced more by the broadband than by the notched noise. In the 1987 model, a larger loudness reduction results in a smaller value of $R$ and hence of the exponent, $p^{\prime}$, used in loudness discrimination $\left(p^{\prime}=.07 R\right)$. If $k$, the constant

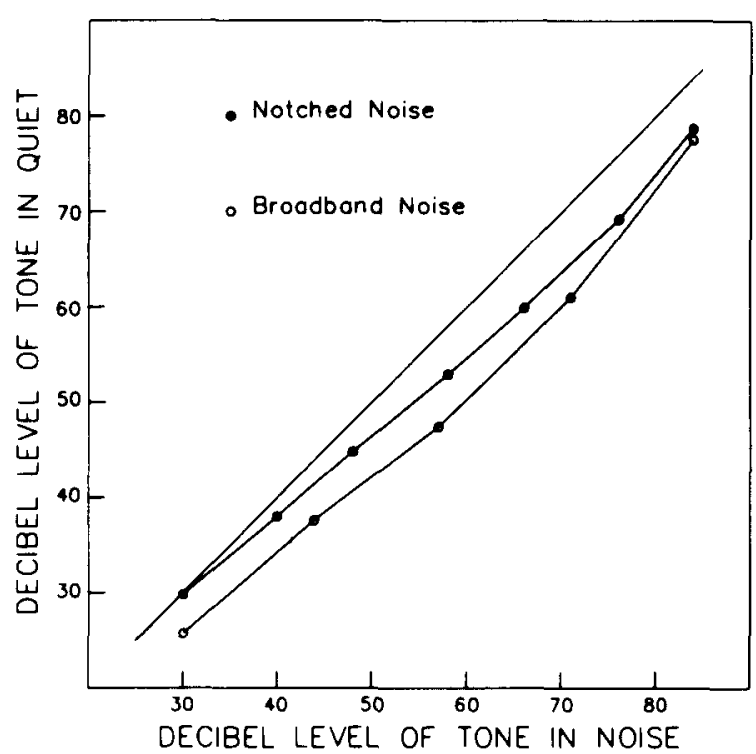

Figure 1. Intensity of tone in quiet that matches the loudness of a tone in notched noise (filled circles, from Schneider \& Parker, 1987 ) and in broadband noise (present data, unfilled circles).

loudness difference at threshold, is the same for notched and broadband noise, then it is easy to see that, for fixed $I_{s}$, to maintain the value of $k, I_{\mathrm{c}}$ must increase if $p^{\prime}$ decreases, because in the model, $k=I_{c}^{P^{\prime}}-I_{s}^{p^{\prime}}$. Therefore, $\Delta I=10 \log \left(I_{\mathrm{c}} / I_{\mathrm{s}}\right)$ should be larger in broadband noise than in notched noise if the model is to relate loudness to loudness discrimination in both notched and broadband noise. However, as Table 1 shows, for Subjects L.T. and R.L., $\Delta / s$ in broadband noise exceed those in notched noise only for the 30-dB standard. For Subject T.T., notched noise provides higher values of $\Delta I$ for standard tone intensities of 71 and $84 \mathrm{~dB}$. Thus, for 10 out of the 15 cases, the obtained value of $\Delta I$ for broadband noise was smaller than, rather than larger than, its value in notched noise. Furthermore, when the parameters of the model were fit to the data (see Schneider \& Parker, 1987 , for details of the fitting procedure), estimates of the exponent, $p$, for the growth of loudness in quiet were .001 , .09 , and .18 for Subjects L.T., R.L., and T.T., respec-

Table 1

Intensity-Increment Thresholds $\left[\Delta I=10 \log \left(I_{c} / I_{s}\right)\right]$ in Notched Noise, Broadband Noise, and Quiet

\begin{tabular}{clccccc}
\hline & & \multicolumn{5}{c}{ Intensity of Standard in Decibels } \\
\cline { 3 - 7 } Subject & Condition & 30 & 44 & 57 & 71 & 84 \\
\hline \multirow{3}{*}{ L.T. } & Notched & 1.85 & 1.80 & 1.87 & 2.31 & 2.13 \\
& Broadband & 2.02 & 1.17 & 1.34 & 1.28 & 1.19 \\
& Quiet & 1.00 & 0.94 & 0.80 & 0.47 & 0.50 \\
& Notched & 1.54 & 1.79 & 1.56 & 1.68 & 1.86 \\
R.L. & Broadband & 1.76 & 1.08 & 1.19 & 1.38 & 0.96 \\
& Quiet & 1.47 & 1.28 & 0.77 & 0.71 & 0.70 \\
& Notched & 1.12 & 1.08 & 0.97 & 2.00 & 1.85 \\
T.T. & Broadband & 1.29 & 1.21 & 1.19 & 1.00 & 1.00 \\
& Quiet & 1.09 & 0.90 & 0.61 & 0.50 & 0.43 \\
\hline
\end{tabular}


tively. Thus, although the degree of fit of the model was a little better for broadband noise, the estimates of the exponent for the growth of loudness in quiet were unreasonably low.

To confirm that the model was unable to arrive at reasonable values of the loudness exponent, $p$, for broadband noise, loudness matches and intensity-discrimination thresholds in broadband noise were obtained from $3 \mathrm{ad}-$ ditional naive subjects. Reasonable fits but unreasonably low estimates of $p$ were again observed for 2 of the 3 subjects. Thus, the model we proposed that managed to describe the relationship between intensity discrimination and loudness for tones in quiet and for tones partially masked by notched noise does not do so for tones partially masked by broadband noise. The present experiment shows that the spectral composition of the masker affects the relationship between loudness and intensity discrimi- nation in ways that cannot be accounted for by the model. Furthermore, we do not see any way in which our model can be extended to incorporate the present results. We hope that other approaches to Fechner's problem will prove more fruitful.

\section{REFERENCES}

Marks, L. E. (1974). On scales of sensation: Prologomena to any future psychophysics that will be able to come forth as science. Perception \& Psychophysics, 16, 358-376.

PARKER, S., \& SCHNEIDER, B. (1980). Loudness and loudness discrimination. Perception \& Psychophysics, 28, 398-406.

SChNeIder, B. A., \& PARKer, S. (1987). Intensity discrimination and loudness for tones in notched noise. Perception \& Psychophysics, 41, 253-261.

(Manuscript received July 18, 1989; accepted for publication August 13, 1989.) 